\title{
Numerical solution of transient temperature for pulsed heating in a single spherical particle model
}

\begin{abstract}
The transient temperature for pulsed heating in a single spherical model has been derived using the Laplace transform algorithm based on Fourier series. We assumed that the substrate is a thermally bad conducting surface and then head conduction at the interface can be neglected. The computation for the transient surface temperature of the particle was performed in such a way as to obtain a straightforward deviation of the analytical solution (12) from the numerical result. It has been proved that for some particles $(\breve{U}<0.005)$ the analytical solution provided a reasonably accurate description of the model, otherwise the numerical solution should be used in the general case.
\end{abstract}

Keyword: Heat conduction; Laplace transform; Particulates; Photothermal wave; Pulse 